\title{
GODAYOL, Pilar; y Taronna, Annarita (eds.). (2018). Foreign Women Authors under Fascism and Francoism: Gender, Translation and Censorship. Newcastle: Cambridge Scholars.
}

TT

ablar de traducción dentro de contextos comparatistas implica apuntar a conversaciones sobre lo que se entiende por ella, incluidas sus posibilidades metafóricas que han sido centrales dentro de ciertas áreas de las ciencias sociales y de los estudios literarios. Asimismo, la traducción ha atravesado permanentemente las discusiones sobre el surgimiento, desarrollo y transformación de la literatura comparada, y sus relaciones con la exploración de las literaturas nacionales y de la literatura mundial, que en cierta medida tocan cuestiones de legitimidad, accesibilidad y los varios "centrismos" que han sido criticados desde espacios como los estudios poscoloniales y los estudios de género. Dentro de estos mismos diálogos, algunos más amables que otros, está, innegablemen-

\author{
JULIA GONSTANTINO REYES \\ Facultad de Filosofía y Letras \\ Universidad Nacional Autónoma de México
}

te, el surgimiento de los Estudios sobre la Traducción dentro de la traductología contemporánea y el planteamiento de su autonomía y de la posibilidad y necesidad de observar y estudiar la traducción per se, a sabiendas de que ese per se ya es suficientemente pantanoso por la polisemia del término traducción y porque se ha convertido en una herramienta teórica, metodológica y crítica para abordar diversos tipos de fenómenos, creaciones e interacciones.

Es dentro de este marco de problemáticas que puede seguir planteando el tema de la traducción que la lectura de Foreign Women Authors under Fascism and Francoism es relevante para varios contextos culturales-nacionales y diversas disciplinas. El libro es resultado del esfuerzo de dos grupos de trabajo, 
el Grup d'Estudis de Gènere: Traducció, Literatura, Història i Comunicació de la Universidad de Vic-Universitat Central de Catalunya y el Archivio di Genere Carla Lonzi de la Universidad de Bari, y del proyecto "Traducción y censura: género e ideología” financiado por el Ministerio de Economíay Competitividad de España. Fue compilado por Pilar Godayol, profesora de traducción de la Universidad de Vic-Universitat Central de Catalunya y coordinadora del Grup d'Estudis de Gènere, y por Annarita Taronna, investigadora de letras inglesas y traducción en el Departamento de Educación, Psicología y Comunicación y coordinadora del Archivio i Genere de la Universidad de Bari Aldo Moro en Italia.

Se trata de nueve ensayos en inglés divididos en dos partes, "Fascism (19221940)” y “Francoism (1939-1975)", cada una precedida de una breve presentación de Taronna y Godayol, respectivamente (la de Godayol traducida por Sheila Waldeck), con una introducción general de las dos traducida por Waldeck que da cuenta del planteamiento de toda la compilación. Esta organización nos permite vislumbrar desde el inicio la claridad conceptual y metodo- lógica del proyecto, pues dentro de lo que hoy en día podemos señalar como una línea historiográfica del giro cultural de los Estudios sobre la Traducción, este libro separa y vincula a la vez dos procesos políticos, sociales y culturales emparentados que tuvieron sus propios mecanismos y repercusiones en sus contextos de producción o impedimento de producción cultural y traductora: el fascismo en Italia y el franquismo en España. Este diálogo de dos tradiciones desde la exploración académica replica, también, el incluido por Godayol entre Giulio Einaudi y Carlos Barral como epítome de la labor editorial solidaria y subversiva que buscaba evadir la censura durante los regímenes totalitarios que les tocó enfrentar. Taronna dice en su presentación de "Fascism (19221940)" que:

[... the research also attempts
to examine censorship as a
multidimensional experience which
depends on the potentially
infinite multiplication of the
interstitial spaces and perspectives
emerging in the gaps between
dominant and subaltern subjects 
(Billiani 2006, 75). By exploring censorial mechanisms in terms of ideology, nationalism, traditionalism, it is possible to fill a historiographical gap by discussing some issues around translation, censorship and the representation of gender identities in Italy's Fascist past and to provide unprecedented insights into translation studies and practices that have reiterated the importance of investigating dominant structures of knowledge and power which are implemented in every translation. (11-12)

La compilación está determinada por otra línea de exploración que multiplica los alcances materiales e ideológicos de la primera: la traducción de algunos textos escritos por mujeres, muchas de ellas conocidas por ser autoras conscientes de las luchas sociales y políticas de las mujeres. Esto hace más complejos los momentos históricos y traductores que aborda el libro porque pone bajo la luz la búsqueda de control y definición estricta de las mujeres durante esos regímenes, la producción y fortalecimiento de estereotipos mediante la literatura y su traducción, y también la búsqueda de transformación de lo anterior a través de la traducción misma mediante la construcción de estrategias subversivas que pudieran resistir los impulsos de las ideologías y regímenes dictatoriales. A la línea historiográfica de los Estudios sobre la Traducción se agrega aquí el enfoque feminista de la traducción, surgido en la década de 1980, al recuperar las voces de algunas autoras, en varios casos la presencia de algunas traductoras, al trabajar con la manipulación textual de representaciones - y de la traducción misma como creación y representación-para lograr conservar o modificar significados, según fuera necesario.

Cada sección se estructura siguiendo una línea que va de los artículos más panorámicos a los estudios de caso específicos, lo cual permite ir construyendo niveles de exposición complementarios que se explican y enriquecen mutuamente en la combinación de aproximaciones teóricas, críticas, históricas y prácticas. La primera sección, dedicada al fascismo en Italia, está formada por "Translating Women Authors under Fascism, Lessons from America" (Valerio Ferme, Universidad de Colorado 
en Boulder), quien mira de manera más o menos general las traducciones al italiano de obras de Louisa May Alcott, Harriet Beecher Stowe, Pearl Buck y Gertrude Stein. Por su parte, Eleonora Federici (Universidad de Nápoles) revisa la existencia e implicaciones de una serie de omisiones y transformaciones en "Whodunit? Agatha Christie's Detective Fiction and the 'Oblique' Translation of Murder on the Orient Express under Fascism”, mientras que Vanessa Leonardi (Universidad de Ferrara) compara analíticamente la primera versión italiana de una novela de Karin Michaëlis, realizada durante el fascismo, con una retraducción de 2005 y con la primera traducción al alemán en "Foreign Women Writers in Fascist Italy: The Case of Danish Bibi and Her Double Censorship", donde se pregunta constantemente por las posibles oscilaciones entre la manipulación textual de quien traduce y la censura institucional. Por último, en "Translating Queerness in Italy's Fascist Past: The Intertwined Stories of Radclyffe Hall, Vita Sackville-West and Virginia Woolf", Taronna muestra la importancia del trabajo de archivo para explorar desde una perspectiva historicista los lazos entre traducción, censura y género que pueden apuntar al poder y presencia de quienes traducen como imprescindibles agentes culturales de resistencia. Puede resumirse el minucioso trabajo hecho en esos artículos $-\mathrm{y}$, en realidad, en toda la compilación- con este comentario de Taronna un poco más centrado en la dimensión concreta de la traducción afectada por la censura:

The close analysis of history, censorship and the translation of foreign women writers through Italy's Fascist past shows that translation may play an important role in provoking a shift in the paradigm when aesthetic criteria of assessment have to come to terms with the rules imposed by publishers' needs and the government's censorship. The fracture of the paradigm here represented by the translation of such writings poses many questions to the reader undermining Fascist authority, and this was not acceptable, since texts needed to support Fascist ideology and values and perpetuate a definite idea of 
"Italianess" and national identity. From this perspective, anything could be changed, scenes or conversations among characterseven if illuminating for the plot's development-could be omitted, foreign words could be deleted and characters could be presented in a totally different way from the original characterization, in their name, nationality, way of speaking and thus narrative function. It was paramount that anything clashing with the Fascist representation of the nation, of Italian identity and culture, of virility and femininity, should be omitted. (10-11)

La sección dedicada a la traducción de textos de mujeres durante el franquismo, presentada por Godayol, sigue la misma estructura que la anterior; no obstante, si en el caso italiano Taronna ofrece una presentación con una visión cultural y crítica amplia y con puntos teóricos claramente identificables, la de Godayol nos lleva de lleno a una serie de apuntes históricos y culturales específicos del franquismo que complementan armónicamente lo expuesto por Taronna. Si bien todos los ensayos están marcados por una visión historiográfica, de archivo y de la traducción como microhistoria, puntos centrales y de enorme relevancia en las discusiones traductológicas actuales, la introducción de Godayol, más que anunciar, es ya muestra de la construcción de esa visión que quedará plenamente ilustrada por ellos. Los alcances y la pertinencia de esa aproximación señalan la forma como la traducción, las traducciones y su circulación dependen de su ubicación espacio temporal en medio de una interacción compleja de sistemas culturales, políticos e históricos. A esto se agrega la manera en que la traducción y las traducciones mismas pueden afectar esos sistemas al introducir, directa u oblicuamente, ideas y propuestas potencialmente desestabilizadoras, e incluso al afectar sistemas próximos, como los de la traducción y publicación en México y Argentina, situación que es mencionada en un par de artículos.

Esta segunda sección está formada por "Catalan Women Translators under Francoism: (Self-)Censorship, Exile, and Silence" de Montserrat Bacardí (Universidad Autónoma de Barcelona) y 
traducido por Fiona Kelso, que discute la existencia de una serie de traductoras catalanas que tuvieron una presencia muy activa durante el primer periodo de la dictadura (1939-1962) cuando las publicaciones catalanas no eran perseguidas, y apunta a momentos posteriores tras la guerra cuando sus traducciones circulaban de manera clandestina. En "Gender, Translation and Censorship in Seix Barral's 'Biblioteca Breve' and 'Biblioteca Formentor' (1955-1975)", Fernando Larraz (Universidad de Alcalá de Henares) recupera la importancia de las colecciones en su dimensión paratextual y como proyectos culturales y políticos que pueden constituir espacios de resistencia a través de la construcción y uso de poder simbólico al conformar un catálogo que incluye, en estos casos, las traducciones de obras de Nadine Gordimer, Margarite Duras, Doris Lessing, Dacia Maraini, Mary McCarthy, Carson McCullers y Nathalie Sarraute. Los tres artículos restantes, " $A$ Vindication of the Rights of Women: The Awaited Right to be Published in Spain" de Carmen Camus Camus (Universidad de Cantabria), "Translating Simone de Beauvoir before the 'Voluntary Consulta- tion"” de Pilar Godayol (Universidad de Vic-Universidad Central de Cataluña) y traducido por Waldeck, y “To Kill a Classic: Harper Lee's Mockingbird and the Spanish Censorhip under Franco" de Cristina Gómez Castro (Universidad de León), son resultado de la combinación de ubicaciones contextuales, investigación histórica de archivo y análisis textual lingüístico-traductológico. El uso conjunto de estas herramientas para la exploración traductológica permite dar sentido a decisiones concretas de traducción -desde qué se decide traducir hasta cómo se logra publicar y difundir, pasando por estrategias y procedimientos de traducción específicos- que explican la relevancia de las obras ya traducidas y su circulación.

La línea historiográfica de esta compilación, que echa mano de los andamiajes de la microhistoria, es desplegada en todos los textos de manera tal que se le observa no sólo como una de las tantas posibilidades de investigación desde los Estudios sobre la Traducción a modo de justificación de la existencia de la disciplina, sino que permite genuinamente observar la riqueza del trabajo interdisciplinario y la relevante función 
protagónica de la traducción y de las traducciones en procesos históricos y sociales. Además de ser en su totalidad una muestra del uso conjunto de metodologías y enfoques teóricos y críticos, otra virtud de esta compilación es que, al reunir los nombres y quehacer de cuatro personas de la academia italiana y cinco de la española, nos recuerda que los Estudios sobre la Traducción tienen que redoblar esfuerzos para fracturar el anglocentrismo académico que los ha caracterizado en las últimas décadas. 